\title{
HIPPARCOS First Results in the Double Star Processing by the FAST Consortium
}

\author{
F. MIGNARD ${ }^{1}$, M. BADIALI ${ }^{2}$, P. L. BERNACCA ${ }^{3}$, H. BERNSTEIN ${ }^{4}$, \\ D. CARDINI ${ }^{2}$, A. EMANUELE ${ }^{2}$, J. L. FALIN ${ }^{1}$, M. FROESCHLÉ ${ }^{1}$, \\ R. HERING ${ }^{4}$, G. PREZIOSO \\ 1 Observatoire de la Côte d'Azur / CERGA, Av. Copernic, F06130 \\ Grasse, France \\ 2 Istituto di Astrofisica Spaziale CNR, Frascati, Rome, Italy \\ 3 Department of Astronomy, University of Padova, Italy \\ 4 Astronomisches Rechen Institut, Heidelberg, Germany \\ 5 Tecnopolis - CSATA, Bari, Italy
}

\begin{abstract}
After the processing of one year of observations carried out by HIPPARCOS it is possible to provide quantitative results as to the number of new double and multiple stars to be detected and the real capacity of this mission to perform relative astrometry on double stars. We present and discuss the methods developed to this end and include the first results concerning the detection statistics and the determination of separation and position angle for double stars. About 16,000 stars have been recognized as non-single, including 9,000 already known as double and mutliple before the mission. Also, a subset of 10,500 stars have been successfully solved for their relative coordinates with an accuracy in the range of 3 to 10 mas.
\end{abstract}

\section{INTRODUCTION}

The capability of the HIPPARCOS mission to add to its main objective of astrometry of single stars, the discovery and measurement of a large number of double and multiple stars, was recognized very early during the mission definition (Lindegren 1979). This has led to the selection of a grid somewhat optimized to this end, without degrading the main mission. A considerable amount of time within the groups responsible for the HIPPARCOS data processing has been devoted since, to devise powerful algorithms in order to retrieve from the counts the astrometric and photometric information pertaining to double and mutliple stars. The procedure can be summarized into three (non-independent) steps, each requiring specific numerical and statistical methods:

- Recognition as early as possible that an HIPPARCOS source is not a single star.

- Photometric analysis of the signal and determination of the magnitude difference between the components.

- Relative astrometry on the sky, giving the geometric description of the system on a plane tangent to the celestial sphere.

The first step allows to flag the stars and avoids the useless and costly processing of single stars in the next two steps. In addition a certain fraction of stars are detected as non-single, but either the separation is too small or the number of observations not large enough to allow the computation of a solution. 
However it is of great scientific value to know that the star is multiple, for example to prepare additional ground-based observations. Finally it is important for the general data processing that only single stars are included in the main reduction chain yielding the HIPPARCOS reference system.

\section{THE HIPPARCOS SIGNAL}

Whenever a star crosses the main grid the counts recorded by the IDT (Image Dissector Tube) are sampled at $1200 \mathrm{~Hz}$ and downlinked to the receiving station. Fourier analysis of short stretches of data results in the determination of five essentially independent signal parameters:

- the mean intensity, which depends mainly on the star magnitude;

- the two amplitudes of modulation which depend on whether the object is a point source or not;

- the two phases of modulation at a given instant which depend on the position of the image within a slit and on its structure.

The phases and modulations are extracted from the modulated signal by fitting the counts collected over a typical duration of 1 second to the five parameter model,

$$
S(t)=I+B+I M \cos (\omega t+\phi)+I N \cos (2 \omega t+\psi)
$$

where $I$ is the total intensity, $B$ the unmodulated background, $M$ and $N$ the modulation coefficients of the first and second harmonic respectively and $\phi$ and $\psi$ the corresponding phases.

For a single star, we have typically $M=0.72$ and $N=0.25$ with a slight dependence on the star color and on the image position on the grid; however the ratio $N / M=I N / I M$ is less variable, over the field and for stars of various colors, than the coefficients taken separately and can be calibrated with a relative accuracy better than 0.005 . Regarding the phases now, for a single star the two phases are not independent but satisfy $\phi-\psi / 2=\delta$ where $\delta$ called the phase difference, is an instrumental parameter easily calibrated about twice a day.

Consider now the extension of this model to a double star. As a result of the linearity of the HIPPARCOS detector the contribution of each star adds as,

$$
S(t)=S_{1}(t)+S_{2}(t)
$$

and gives eventually a signal model similar to Eq. 1, without the constraints between the modulation coefficients and phases, which are specific to single stars. Incidentally the HIPPARCOS magnitude $H_{p}$ to which we will refer to below is defined by

$$
H_{p}=-2.5 \log _{10}\left(\frac{I}{I_{0}}\right)
$$

\section{STATISTICAL TESTING}

Some 14,000 double and multiple stars are already flagged in the Input Catalogue; they are either considered as combined pairs, in which case a single entry 
contains the information on the system, or as detached systems with at least two separate entries in the Catalogue (Dommanget et al. 1989). Most of the selected binaries have a separation between the two components larger than 1", much larger than the detection threshold of HIPPARCOS. Hence, it is absolutely necessary to rely on an internal recognition method, to supplement the information available in the Input Catalogue. The double star testing consists in determining whether the actual signal departs significantly from the single star model and in concluding safely that this is due to a multiple star and not to a statistical fluctuation. We wish to detect as many as possible multiple stars without falsely including too many singles. As ever in statistical testing we face the trade-off between errors of the first and second kind. The thresholds have been set both from theoretical consideration and also by trial and errors on stars whose nature was known. We have built essentially three tests to accept or reject the null hypothesis

$$
H_{0}=\text { The star under consideration is single. }
$$

The properties and setting up of these tests are described in Mignard et al. (1992b), so we restrict here to giving their main features. As said before the phase shift between the first and second harmonic and the two modulation coefficients are fairly well known for single stars, as well their statistical distributions. Let $M_{0}$ and $N_{0}$ be the single star modulation coefficients. We consider the following combinations of the phases and amplitudes:

$$
R=\frac{N}{M} / \frac{N_{0}}{M_{0}}
$$

called the amplitude ratio and scaled to the single star value. Whenever $R \approx 1$ the star is likely to be single.

Then let

$$
\delta=\phi-\psi / 2
$$

be the phase difference corrected from all the known instrumental effects, in such a way that for a single star $\delta$ should be zero. The typical precision on the phase $\phi$ for a single star of $9 \mathrm{mag}$ is $10 \mathrm{mas}$, and the instrument corrections are of the order of 15 mas, with color dependence.

Finally, we set the third test with

$$
\Delta=-2.5 \log _{10}\left(\frac{M \cdot M_{0}+N \cdot N_{0}}{M_{0}^{2}+N_{0}^{2}}\right) .
$$

It is called the magnitude test, since it is intimately connected to the HIPPARCOS photometry (Mignard et al. 1992a). The expectation of this quantity is also zero for a single star and a positive value signals a multiple star.

Figure 1 shows the values of $R, \delta$ for a double star as a function of the separation projected along the normal to the grid slits and of the magnitude difference between the primary and secondary of the binary. We see that the amplitude ratio takes the single star value for projected separation of $120^{\circ}$ and $240^{\circ}$, or approximately 0.4 and $0 " .8$ for a gridstep of $1^{\prime \prime} .208$. As expected, we also find that a multiple star cannot be recognized in the signal whenever the 

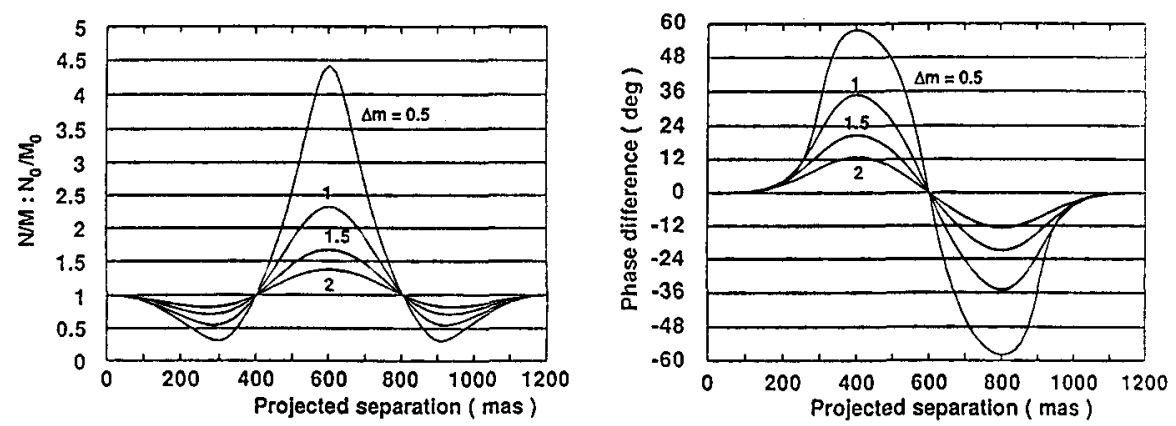

FIGURE 1. Departure of the signal of a double star from that of a single star.

(left) Ratio of the amplitude of the second to the first harmonic.

(right) Phase difference between the two harmonics; $1^{\circ} \approx 3$ mas.

projected separation is an integral number of gridsteps. The phase difference does not allow to detect double stars with separation of 0 ".6, modulo half a gridstep. Fortunately the two regions of inefficiency do not overlap, and there is always at least one of the two tests which applies. Finally the magnitude test $\Delta$ proves to be efficient almost everywhere.

The departure of $R, \delta$ and $\Delta$ from their single star values has to be assessed against the natural scattering exhibited by a single star, and this is a function of the magnitude. For large sigmas, it is impossible to decide whether the departure from the single star value results from chance fluctuation about the average or from the presence of a companion close to the target star. Without entering into too much detail, it is enough to know that for a typical HIPPARCOS star of $9 \mathrm{mag}$, the statistical fluctuations, for data accumulated over a full transit on the grid, make $R$ fall in the range $[0.95,1.05], \delta$ in $\left[-3^{\circ}, 3^{\circ}\right]$ while $\Delta$ has fluctuations less than $0.05 \mathrm{mag}$. These figures are typical values applicable to the bulk of the HIPPARCOS Catalogue, that is to say for a star of magnitude 8.5. The power of the tests degrades with increasing magnitude. By imposing a detection margin of the order of two sigmas, one sees immediately on the plots that double stars are be recognized provided the projected separation exceeds 0 ". 15 for primary and secondary equally bright. The performances degrade, but slowly, with increasing magnitude difference.

We have analysed the power of these tests on a set of stars known from the HIPPARCOS Input Catalogue to be double, with ground-based values of the separation and magnitude difference. According to the orientation of the scanning circle with respect the radius vector between the primary and secondary, the projected separation may be negligible or as large as the true separation. Therefore a star is detected as non-single provided it passes the test at least once. We have studied the efficiency rate by counting the number of known binaries detected as a function of the separation and magnitude difference. Results 


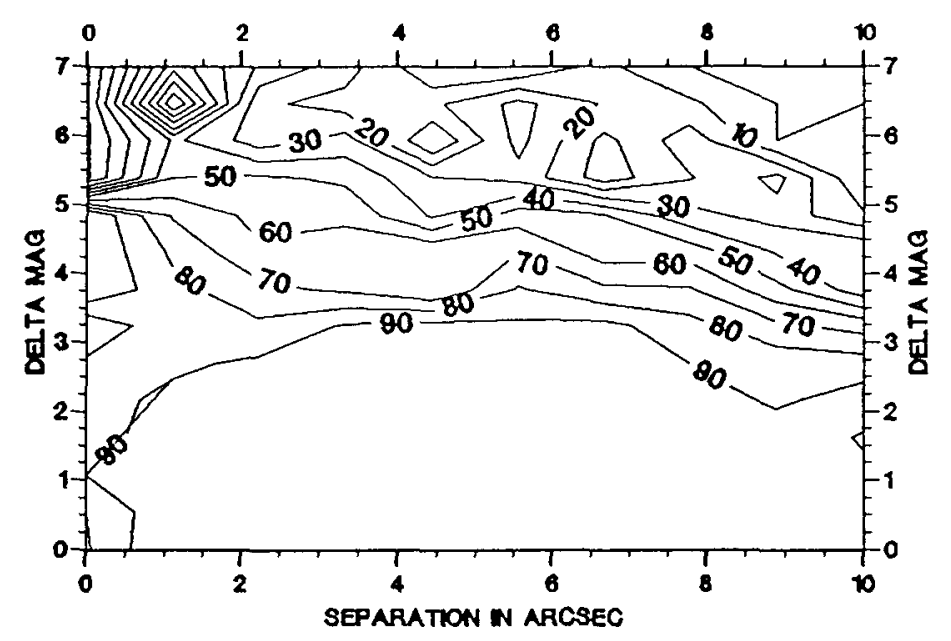

FIGURE 2. Recognition rate in percent of known double stars as a function of the separation and magnitude difference.

shown with contour lines in Figure 2 indicate that the detection is very efficient in a well delimited zone with $\Delta m<4$, with apparently no marked effect with the separation. Unfortunately, the small number of close binary stars available in the Input Catalogue does not allow to refine the analysis to separations less than 0.2 . Nonetheless, we feel confident that the detection extends down to separations of $0 " 15$. This confidence rests upon the recognition of the few close binary stars in the Input Catalogue and on the astrometric solution presented in the next section precisely for close binaries discovered with the tests.

Altogether we have processed data amounting to one year of obervation. On the average a specific star has crossed one of the field of view of HIPPARCOS 25 times, although this number suffers considerable variation between zero, a handful among the 120,000 programme stars have not been sucessfully observed, to 150 for the most observed. The overall statisics of detection are shown in Table 1. About 7,000 stars, given as single in the Input Catalogue are seen at least double by HIPPARCOS. This does not actually mean that all these stars are new double, because the flagging in the Input Catalogue is probably not $100 \%$ complete with respect to known double and multiple stars. In addition there is another set of 4,500 single stars in the Input Catalogue which are marginally detected as double, but with too small a significance level to regard this detection as final.

\section{ASTROMETRIC SOLUTION}

The parameters of the double star on the sky are the separation and the position angle, or equivalently the relative cartesian coordinates on the tangent plane. The HIPPARCOS observations are by nature uni-directional, providing 
TABLE 1. Overall double and multiple stars detection efficiency. The first line refers to the Input data and the leftmost column to the processing.

\begin{tabular}{|lrrrr|}
\hline & Single & $\begin{array}{c}\text { Double } \\
\left(\rho<10^{\prime \prime}\right)\end{array}$ & $\begin{array}{r}\text { Double } \\
\left(\rho>10^{\prime \prime}\right)\end{array}$ & Total \\
\hline Detected as single & 91,000 & 4,100 & 1,100 & 96,200 \\
Dubious detection & 4,700 & 700 & 500 & 5,900 \\
Detected as non-single & 6,800 & 7,800 & 1,500 & 16,100 \\
\hline Total & 102,500 & 12,600 & 3,100 & 118,200 \\
\hline
\end{tabular}

at each star crossing information along the scanning direction. In addition this information is obtained within one gridstep, that is to say projected separations are known to an arbitrary integer number of gridsteps of $1^{\prime \prime} .208$. Therefore the astrometric solution for double stars requires that many observations covering various orientations of the scan circle be brought together to retrieve an unambiguous solution. Although, in principle three well-separated observations are sufficient for this purpose, experiments have shown that at least five such observations are needed to discriminate the true solution from spurious ones.

For a double star without significant orbital motion during the mission lifetime, the double star parameters are constant. If $\rho, \theta$ are the true separation and position angle we have the cartesian coordinates (with $\mathrm{X}$ along the latitude small circle):

$$
\begin{aligned}
& X=\rho \sin \theta \\
& Y=\rho \cos \theta
\end{aligned}
$$

while the projected separation on the scanning circle is:

$$
\Delta S=X \sin \gamma+Y \cos \gamma
$$

where $\gamma$ is the position angle of the scan direction at the time of observation. This separation is just the opposite of the projected separation on the grid, because of the orientation of the $G$-axis on the grid, and is linked to the phase difference $\alpha$ by:

$$
\begin{aligned}
& \cos \alpha=\cos (2 \pi \Delta S) \\
& \sin \alpha=\sin (2 \pi \Delta S)
\end{aligned}
$$

which is eventually related to the unknowns $X, Y$ by

$$
\begin{aligned}
\cos \alpha & =\cos [2 \pi(X \sin \gamma+Y \cos \gamma)] \\
\sin \alpha & =\sin [2 \pi(X \sin \gamma+Y \cos \gamma)] .
\end{aligned}
$$

Equations 9 set the observation model, with $\alpha$ the observed quantity determined at each transit and $\gamma$ computed from the attitude and the star coordinates. The main problem with this model lies in the grid step ambiguities: the same phase 


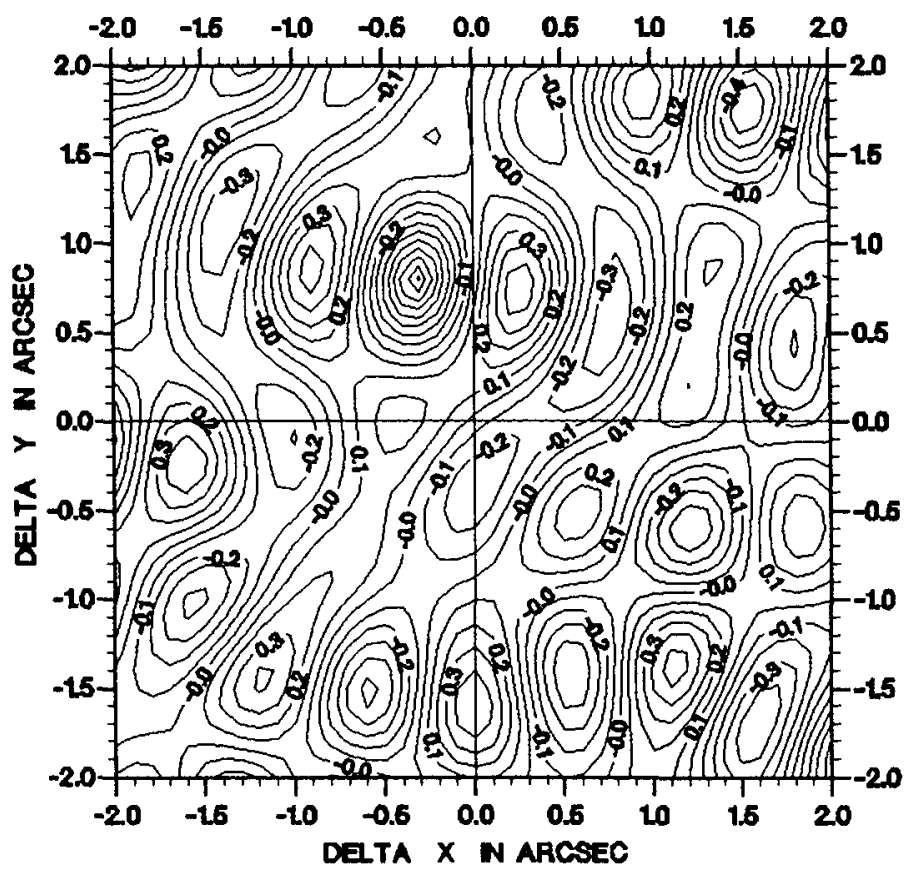

FIGURE 3. Behaviour of the normalized $\chi^{2}$ function used to fit HIPPARCOS observations to a double star model. The center of the diagram is the a priori position of the secondary with respect to the primary. The solution is located at the minimum $\chi^{2}$.

difference $\alpha$ may correspond to projected separations $\Delta S$ differing by an integral number of gridsteps which has to be determined. So we add to each phase difference by a trial-and-error algorithm $2 \pi, 4 \pi, \cdots$, until we reach the best consistency between the observation equations and the model. The goodnessof-fit is measured by the normalized $\chi^{2}$,

$$
U=\sum_{i=1}^{i=n}-w_{i} \cos \left(a_{i} X+b_{i} Y-\alpha_{i}\right)
$$

where $a_{i}=-2 \pi \sin \gamma_{i}, b_{i}=-2 \pi \cos \gamma_{i}$, and $w_{i}$ is the weight applied to observation $i$, with $\sum_{i=1}^{i=n} w_{i}=1$. For a perfect fit one would find $U=-1$; The minimum of $U$ supplies the value of $X$ and $Y$ corresponding to the best fit to the observations.

A typical function $U(X, Y)$ is displayed in Figure 3. The solution retained corresponds to the bottom of the deepest hole in the plane $X, Y$ just above the center on the left. It is clear that the optimization problem is not a trivial one, as many secondary minima appear in the plot, some nearly as deep as the deepest. The depth of the main hole is not constant, and depends on the star magnitude and on the quality of the projected separation obtained on each scan. Thus there is no universal threshold to tell the search algorithm how to locate a small 


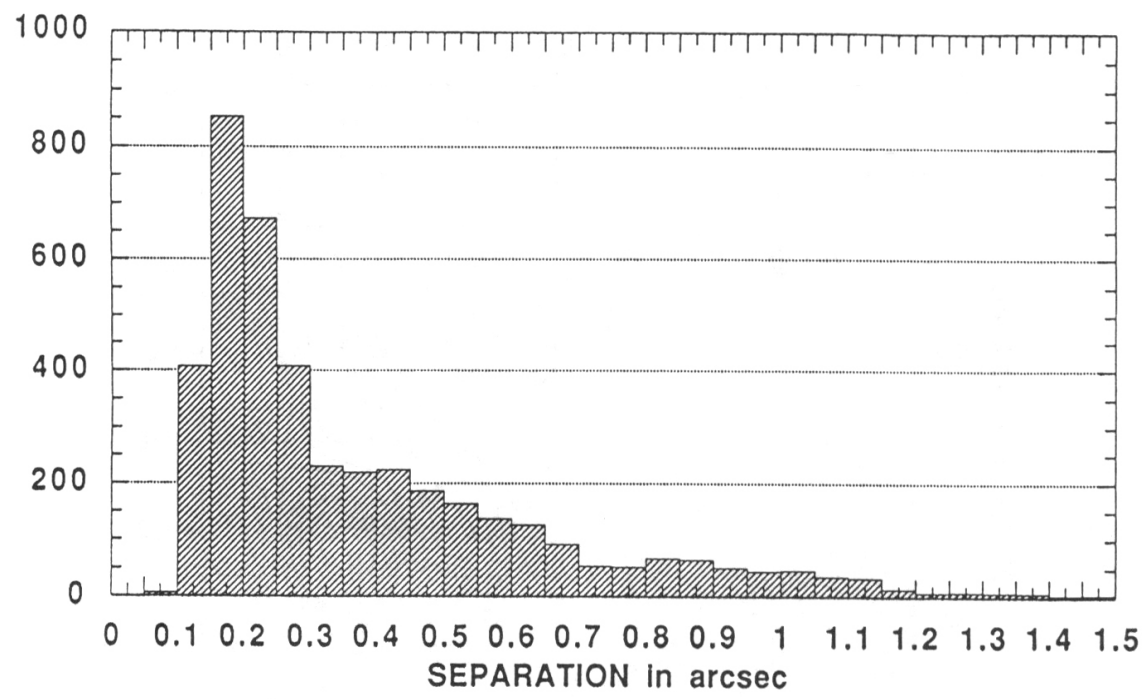

FIGURE 4. Distribution of the separations in the astrometric solution applied to newly recognized double stars.

region in the plane $(X, Y)$ where the deepest hole lies. As the reader can see, the holes appear more or less regularly distributed on a two-dimensional lattice with a period of the order of the second of arc. The mesh-size of the search algorithm needs to be much smaller than this typical size to have a chance of going close to the minima. The algorithm goes to the solution in two steps: first, one tries to find the proper hole, with the help, when available, of the a priori astrometric information on the double star; then a steepest descent followed by a rapidly converging second-order method determines the minimum.

Detail on the procedure and its accuracy are found in Mignard (1988) and Mignard et al. (1989). If the actual model is $X(t), Y(t)$ additional parameters (first, second derivatives, orbital parameters) are added to the model and adjusted to the observations. Finally it must be noted that the formal precision is a measure of the shape of the surface $U(X, Y)$ in the vicinity of the solution. A smooth minimum, will not allow to locate accurately its coordinates, whereas a well defined minimum gives accurate coordinates. But one must be aware that another kind of error is likely to occur, unusual in astronomy. It may happen that the minimum found is not the right one, as several set of integers end up with a satisfactory fit. In this case the astrometric parameters are wrong by about one arcsec, although the formal precision is of the order of 0 ".005. In principle these bad circumstances should disappear with the increasing number of scans in the same way as in a diffraction grating with many slits, the secondary minima vanish with increasing number of slits.

Over one year of processing, as said above, about 16,000 stars were detected as double or multiple. Among this set only 13,000 were retained for search of an astrometric solution on the basis of the number of scans available. The algorithm 
founds a satisfactory solution for about 10,500 while for the other 2,500 the convergence was not achieved or the coordinates we get were clearly wrong. For example, an unknown double star is not likely to be found with a separation as large as $5^{\prime \prime}$ and must be rejected as spurious solution. The distribution of the measured separations for 3,500 stars newly recognized as double by HIPPARCOS is shown in Figure 4. It is no surprise that these separations are rather small compared to the average separation of visual double stars. This strengthens the fact that these numbers are realistic and not simple numerical artifacts. The level of confidence will increase steadily with the inclusion of new observations carried out by the satellite with the minimum of the $U(X, Y)$ function becoming more conspicuous. For the 7,000 stars with a ground-based separation, our solution does not show any systematic difference between HIPPARCOS and earth-based astrometry. However the HIPPARCOS precision is much better, of the order of $6-8$ mas for most stars.

\section{CONCLUSION}

After a reduction covering only one year of data we can draw already interesting conclusions:

- The algorithms, defined and prepared before the mission, work as expected on the real data.

- It is likely that about 8,000 to 10,000 new double stars will be recognized by HIPPARCOS, down to a separation of $0^{\prime \prime} .12$ and relative astrometric parameters will be obtained for stars with separation larger than 0 ".15.

- As we hope to get three more years of data, one may envision a final accuracy in relative positions of a few milliarcsec for most double stars with no significant orbital motion. For the other, a determination of a uniform or accelerated motion will be achievable. The possibility of computing an orbit for the fastest remains a challenge.

\section{REFERENCES}

Dommanget, J. 1989, "Double Stars", in The HIPPARCOS Mission, ESA report SP 1111, III, 149

Lindegren, L. 1979, Coll. European Satellite Astrometry, eds. C. Barbieri and P.L. Bernacca, p. 117

Mignard, F., Borriello, L., Kovalevsky, J., Prezioso, N., \& Bernacca, P.L. 1989, “Double Star Reduction in FAST", in The HIPPARCOS Mission, ESA report SP 1111, III, p. 243

Mignard, F., Froeschlé, M., \&Falin, J.L. 1988, "Photometry with IDT II: Double stars", in Proc. of the Bari coll. Processing of Scientific Data from HIPPARCOS, Third FAST Thinkshop, eds. P.L. Bernacca and J. Kovalevsky, p. 263

Mignard, F., Froeschle, M., \& Falin, J.L. 1992a, "HIPPARCOS Photometry: Main Mission", $A B A$, in press

Mignard, F., Froeschlé, M., Badiali, M., Cardini, D., Emanuelle, A., Falin, J.L., \& Kovalevsky, J. 1992b, "HIPPARCOS double star recognition and processing", $A \mathcal{E} A$, in press 